\title{
An ultrafast laser micromachined broadband terahertz frequency selective surface
}

\author{
S SASI PRINCY ${ }^{1, *} \mathbb{D}$, B S SREEJA ${ }^{1}$, E MANIKANDAN $^{1}$, S RADHA $^{1}$, RAVI NATHURAM BATHE $^{2}$, \\ RAVIKUMAR JAIN ${ }^{3}$ and S S PRABHU ${ }^{3}$ \\ ${ }^{1}$ Department of Electronics and Communication Engineering, SSN College of Engineering, Chennai, India \\ ${ }^{2}$ Center for Laser Processing of Materials, ARCI, Hyderabad, India \\ ${ }^{3}$ Department of Condensed Matter Physics and Materials Science, Tata Institute of Fundamental Research, Mumbai, India \\ *Author for correspondence (sasiprincys@ssn.edu.in)
}

MS received 17 November 2018; accepted 3 March 2019; published online 13 May 2019

\begin{abstract}
The purpose of this paper is to focus on the frequency selective surface (FSS) filter structure which exhibits a broadband frequency response operating in the terahertz $(\mathrm{THz})$ frequency regime. The achievement of the broadband frequency response has been accomplished by means of only invoking the FSS structure excluding the presence of a ground plane and also excluding the multilayered FSS technique. The FSS filter is simulated using CST Microwave Studio. This simulation has been carried out using Teflon as the substrate which is a dielectric over which the conductive material gold with a thickness of $2 \mu \mathrm{m}$ is coated in which the FSS filter structure is designed with periodic hexagonal aperture unit cells. The structure of the proposed frequency selective surface filter helps in achieving a broadband frequency response with a bandwidth of $300 \mathrm{GHz}$ centred at $0.36 \mathrm{THz}$. The designed FSS structure is a bandpass filter and it exhibits the most imperative properties of angle resolvability and polarization insensitivity. The fabrication of the same has been carried out using the laser micromachining process.
\end{abstract}

Keywords. Terahertz (THz); frequency selective surface (FSS); band-pass filter; laser micromachining.

\section{Introduction}

In the recent era, the terahertz $(\mathrm{THz})$ frequency band has been the band of interest since its pros are much higher than the other frequency bands which are there in the already prevailing applications. The applications like $\mathrm{THz}$ imaging and sensing, material characterization, space science and communication are emerging which involve the $\mathrm{THz}$ frequency band as the band of operation. This became an insight for the researchers to pursue attractive research in this area. The components and the modules which are essential for operating in the $\mathrm{THz}$ regime are still insufficient which acts as the barrier for exploring the $\mathrm{THz}$ frequency band. In the wireless communication domain, one of the vital components is the filter. Factors like high-cost of fabrication and degradation due to scratch and dust at the THz frequency are the issues to be considered while designing the modules for operation at the $\mathrm{THz}$ frequency band. The commercial and military applications also find the $\mathrm{THz}$ frequency band suitable for operation in remote sensing and home security application area.

The frequency selective surface (FSS) filters can be of one dimensional or two dimensional structures, whose filtering operation performance relies on the physical properties of the material used and their physical dimensional geometry. These FSS filters can be integrated into spatial communication, ground communication and airborne platforms.
A multilayered FSS and asymmetrical electromagnetic structured FSS have been used for achieving a broadband and multiband frequency response $[1,2]$. These FSS filters are being invoked using different substrates of sapphire and lowloss cyclo olefin polymer Zeonor for the purpose of achieving high-transmission which enables the FSS filters to be more pertinent for use in the $\mathrm{THz}$ frequency regime $[3,4]$.

The need for environmental monitoring has been inevitable in recent years and the process of monitoring the clouds and other greenhouse gases and their effect on the environment had been a tougher job. When the monitoring process has been carried out by employing modules in the space vehicle, many remarkable criteria of the module must be keenly taken care, i.e., being resistant to the harsh thermal environment, being in the micro-scale range, etc. Therefore, the frequency selective surface filter working in the $\mathrm{THz}$ regime has been of great interest because of its tendency to allow penetration and also being capable of observing the clouds in the environment. This paves the way towards the evolution of remote sensing technology. The factor of polarization insensitivity being less sensitive to the oblique angle of incidence plays a major role in the FSS filter structure that is being used in the remote sensing and spatial applications since it may involve a rotation of $360^{\circ}$. The transmission response of the FSS filter structure is significant as it shows the front-end receiver sensitivity invoking the FSS filter, possessing very low 


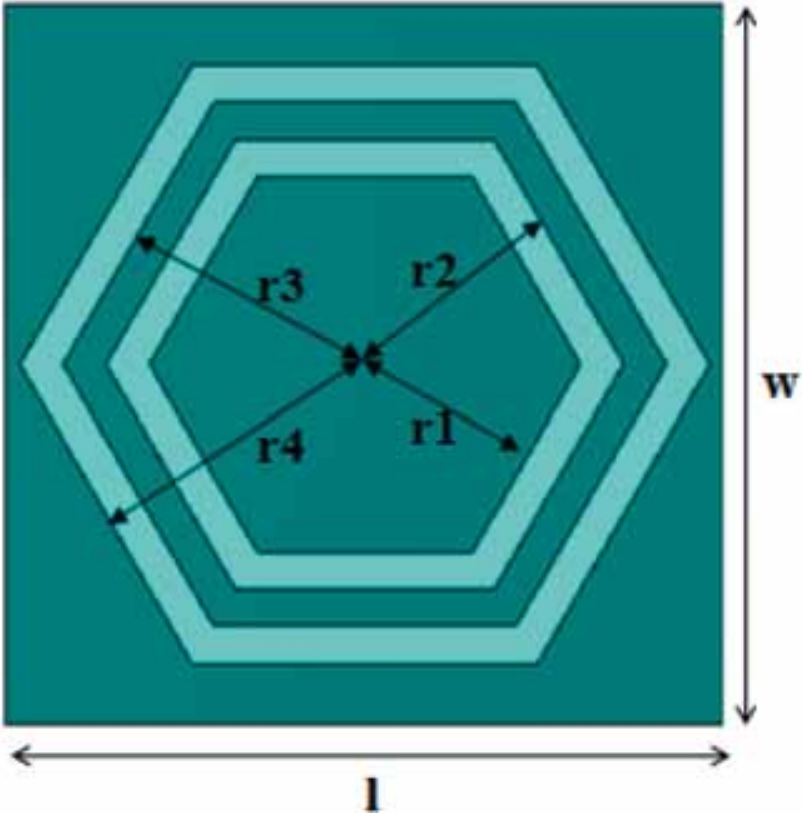

Figure 1. Schematic of the two-concentric hexagonal slot FSS filter structure.

Table 1. Dimensional specifications of the FSS filter structure.

\begin{tabular}{lc} 
Parameters & Dimensions $(\mu \mathrm{m})$ \\
\hline$r_{1}$ & 150 \\
$r_{2}$ & 180 \\
$r_{3}$ & 210 \\
$r_{4}$ & 240 \\
$l$ & 500 \\
$w$ & 500 \\
\hline
\end{tabular}

insertion loss which can be implied in remote sensing and spatial applications $[5,6]$.

Tunability and reconfigurability are achieved by means of invoking FSS and phase changing materials along with vanadium oxide [7] exhibiting the desired $\mathrm{THz}$ frequency response. The conventional methods of achieving the broadband frequency response are by means of invoking the ground plane or defected ground structure [8-10] or multilayered FSS design [11]. The major problem associated with these devices is the fabrication of microscale features on the target materials. This paper addresses this issue by utilizing ultrafast laser ablation which produces the desired structure without any damage to the substrate with the absence of heat affected area compared to nanosecond laser ablation [12].

In this paper, the design of a FSS filter structure exhibiting a broad-band frequency response has been focused. The design of a two-concentric hexagonal slot filter structure has been carried out aiming at a broadband frequency response, but it resulted in an additional resonance in between, exhibiting a dual-band frequency response. For consummating the aim of the broadband frequency response, the two-concentric

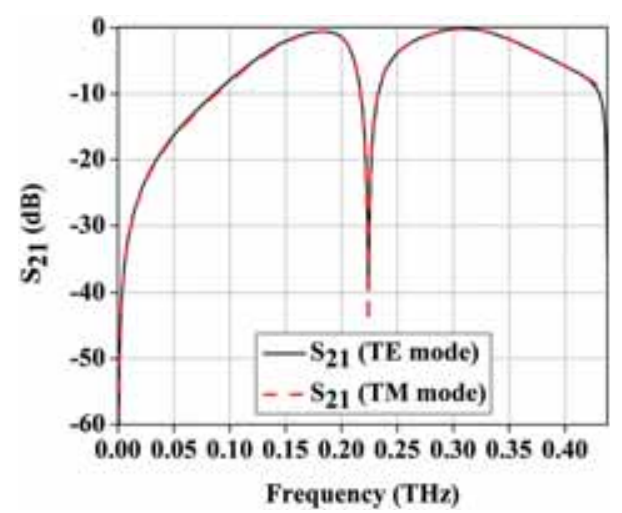

Figure 2. $S_{21}$ of the FSS two-concentric hexagonal slot FSS structure.

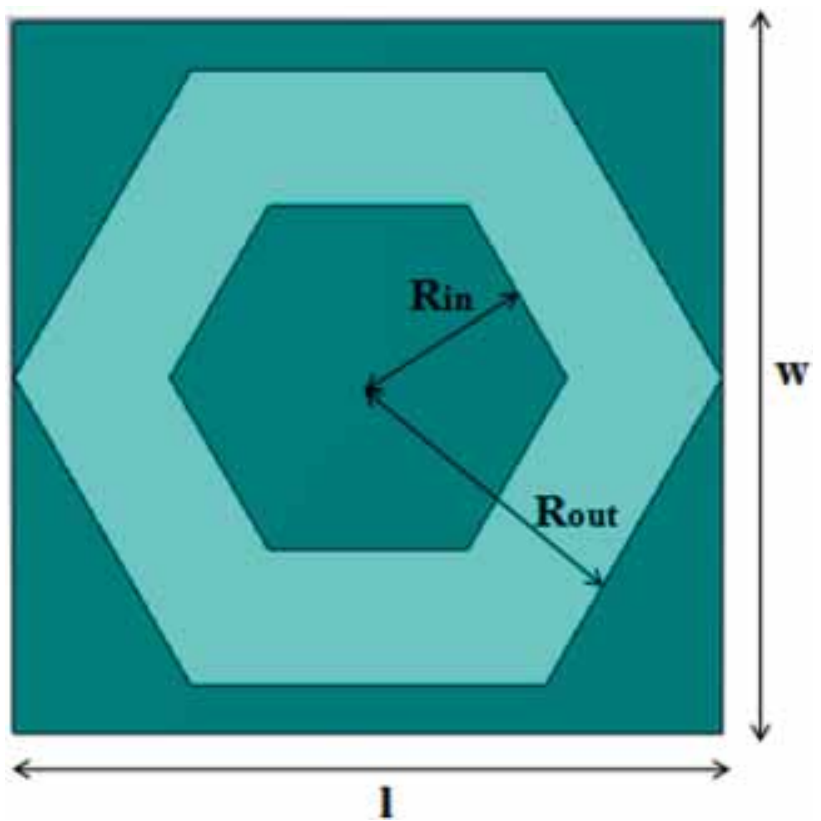

Figure 3. Schematic of the broadband FSS filter structure.

hexagonal slot dimensions were altered in terms of its width leading to the metal present between the two-concentric hexagons being etched as well, forming a single periodic hexagonal aperture unit cell. The single hexagonal slot aperture unit cell FSS filter structure achieved a broadband frequency response with a bandwidth of $300 \mathrm{GHz}$ centred at $0.36 \mathrm{THz}$. Thus it is substantiating the fact that by varying the geometrical parameters of the structure, we can obtain different resonances according to the requirement. The momentous feature of this work is to obtain broadband filtering characteristics using a simple structure. This in turn eases the fabrication process which can be performed by employing a laser micromachining process. This is a direct writing technique which avoids the development of any kind of mask for the fabrication compared to the conventional lithography technique. The femtosecond laser has been invoked for 
Table 2. Design specifications of the broadband FSS filter structure.

\begin{tabular}{lc} 
Parameters & Dimensions $(\mu \mathrm{m})$ \\
\hline$R_{\text {in }}\left(r_{1}-10 \mu \mathrm{m}\right)$ & 140 \\
$R_{\text {out }}\left(\mathrm{r}_{4}+10 \mu \mathrm{m}\right)$ & 250 \\
$l$ & 500 \\
$w$ & 500 \\
\hline
\end{tabular}

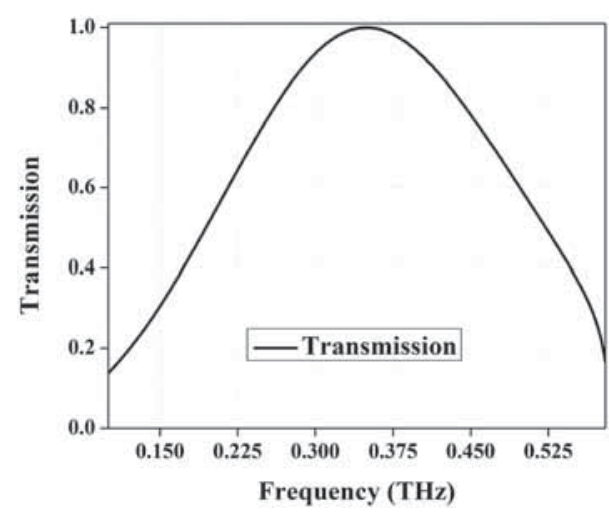

Figure 4. Transmission in decibel.

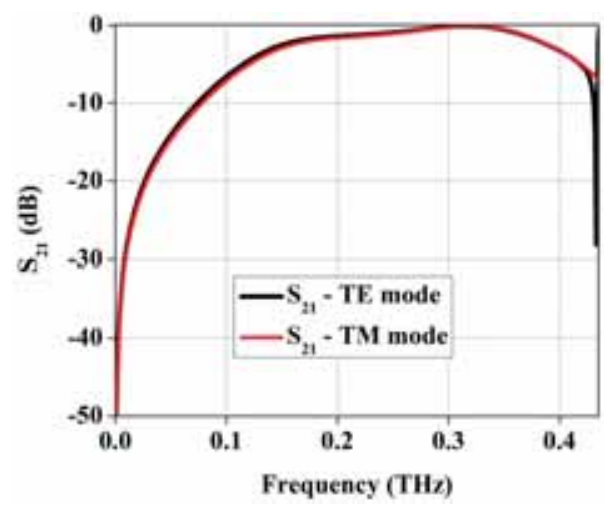

Figure 5. Transmission coefficient, $S_{21}$ for TE and TM modes.

the process of fabrication which is ultrafast and therefore a precise structure has been achieved.

\section{Design methodology}

The frequency selective filter structure involves the shape of two-concentric hexagonal slots. The filter has been designed using a Teflon substrate with a thickness of $250 \mu \mathrm{m}$. The Teflon substrate has been coated with a conducting material gold with a thickness of $2 \mu \mathrm{m}$ in which the frequency selective surface filter has been engraved.

The FSS filter structure involves two-concentric hexagonal shaped metal slots and is shown in figure 1. The hexagonal slot 1 has the inner radius of $150 \mu \mathrm{m}$ and the outer radius of

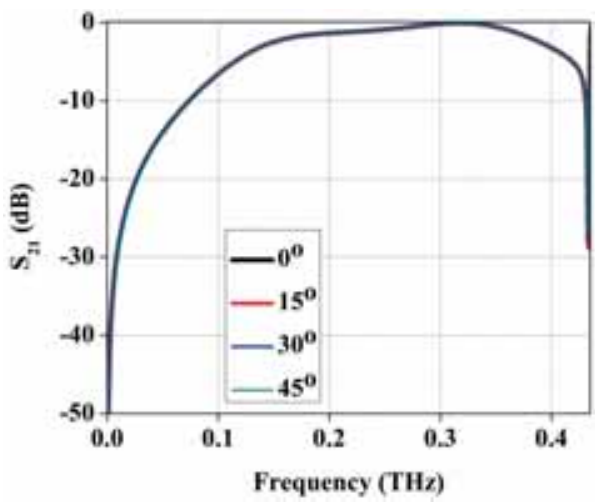

Figure 6. Transmission coefficient, $S_{21}$ for different incident angles.

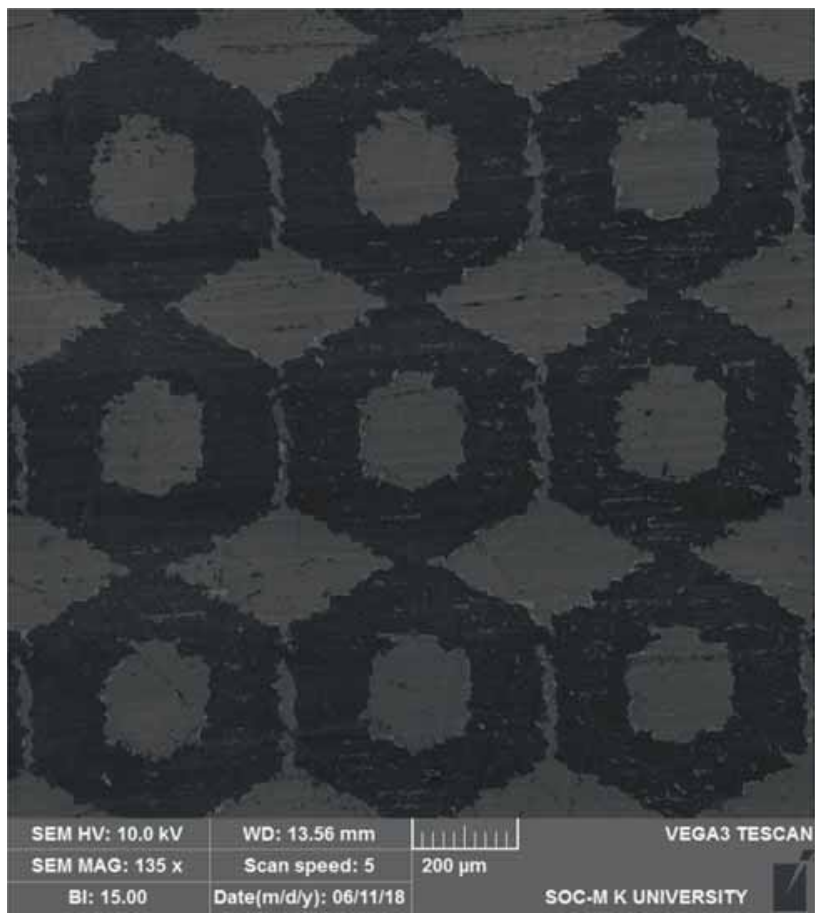

Figure 7. SEM image of the fabricated FSS filter.

$180 \mu \mathrm{m}$. The hexagonal slot 2 has the inner radius of $210 \mu \mathrm{m}$ and the outer radius of $240 \mu \mathrm{m}$. In a unit cell, the two created hexagonal slots produce two hexagons $\mathrm{A}$ and $\mathrm{B}$. The first hexagon $\mathrm{A}$ is inside the hexagonal slot 1 and it has an inner radius of $0 \mu \mathrm{m}$ and an outer radius of $150 \mu \mathrm{m}$. The second hexagon $B$ is present in between the hexagonal slots 1 and 2 and it has an inner radius of $180 \mu \mathrm{m}$ and an outer radius of $210 \mu \mathrm{m}$. Table 1 shows the geometrical dimensions of the designed FSS filter structure.

The two-concentric hexagonal slot FSS filter structure aiming at a broad-band frequency response has been simulated using full-wave EM simulation software CST Microwave Studio. The frequency solver settings are depicted as follows: unit cell boundary conditions along the lateral directions, open 
(add space) boundary conditions along the $z$-direction and a tetrahedral mesh. The ports for the frequency selective surface filter are the wave ports which are automatically added along the direction of normal incidence to the substrate. The gold coated layer is modelled as lossy gold with a conductivity, $\sigma=4.56 \times 10^{7} \mathrm{~S} \mathrm{~cm}^{-1}$. The substrate has a loss tangent value, $\tan \delta=0.0002$ and dielectric constant, $\varepsilon=2.1$.

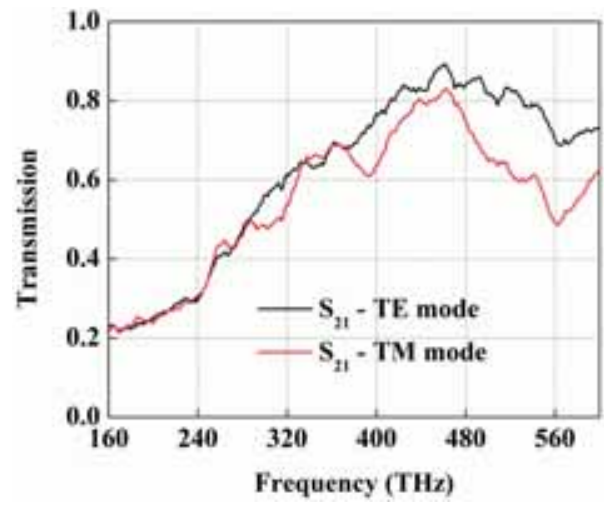

Figure 8. Measured transmission coefficient, $S_{21}$.
The outcome i.e., the transmission coefficient, $S_{21}$, achieved from the designed FSS filter structure is a dual-band response. The obtained dual-band response is depicted in figure 2 . The centre frequency of the obtained dual band is at 0.18 and $0.3 \mathrm{THz}$ and the bandwidth of it is 0.07 and $0.11 \mathrm{THz}$. The frequency at which both the bands are being separated is at $0.24 \mathrm{THz}$.

Still, the focus of achieving a broadband frequency response has been pursued. The designed FSS filter structure has been altered in terms of the width of the two-concentric hexagonal slots. The altered dimensional specifications i.e., by widening the widths of the two-concentric hexagonal slots, the resonance band at the frequency of $0.24 \mathrm{THz}$ has been reduced in steps and the bandwidth has started to become wider. Eventually, for the dimensions when the two-concentric hexagons are merged by means of etching the whole metal strip present between the two slots, the resonance at $0.24 \mathrm{THz}$ disappeared exhibiting a broadband frequency response with a bandwidth of $300 \mathrm{GHz}$. The dimensions of the wide hexagonal slot achieving the aimed broad-band frequency response are: the inner radius is $R_{\text {in }}=r_{1}-10 \mu \mathrm{m}$ and outer radius is $R_{\text {out }}=r_{4}+10 \mu \mathrm{m}$. The final geometric specifications of the FSS filter structure exhibiting a broad-band response is depicted in figure 3 and the values are stated in table 2.
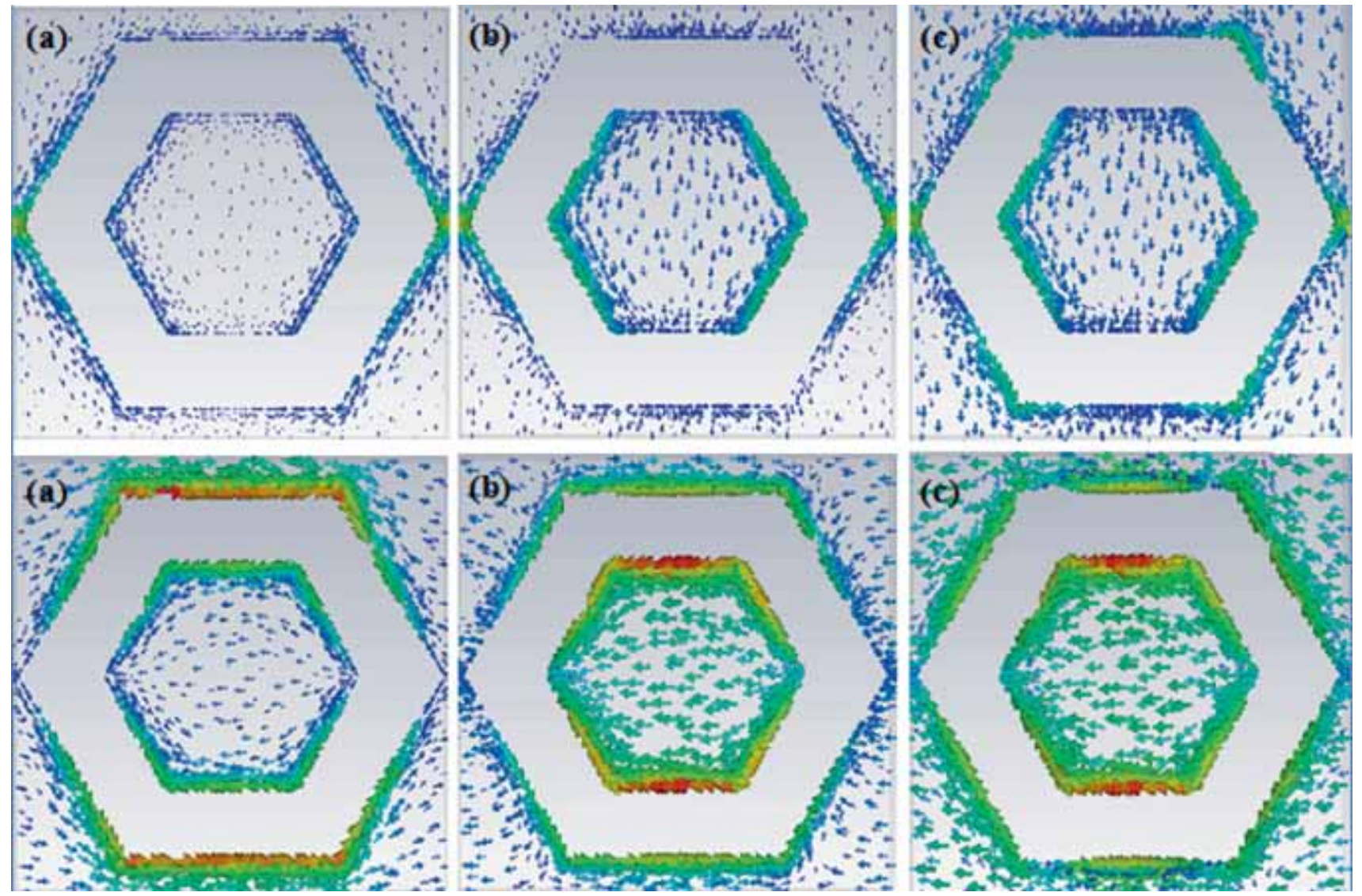

Figure 9. TE mode and TM mode surface current distribution at the three frequencies: (a) $0.2 \mathrm{THz},(\mathbf{b}) 0.36 \mathrm{THz}$ and (c) $0.52 \mathrm{THz}$. 

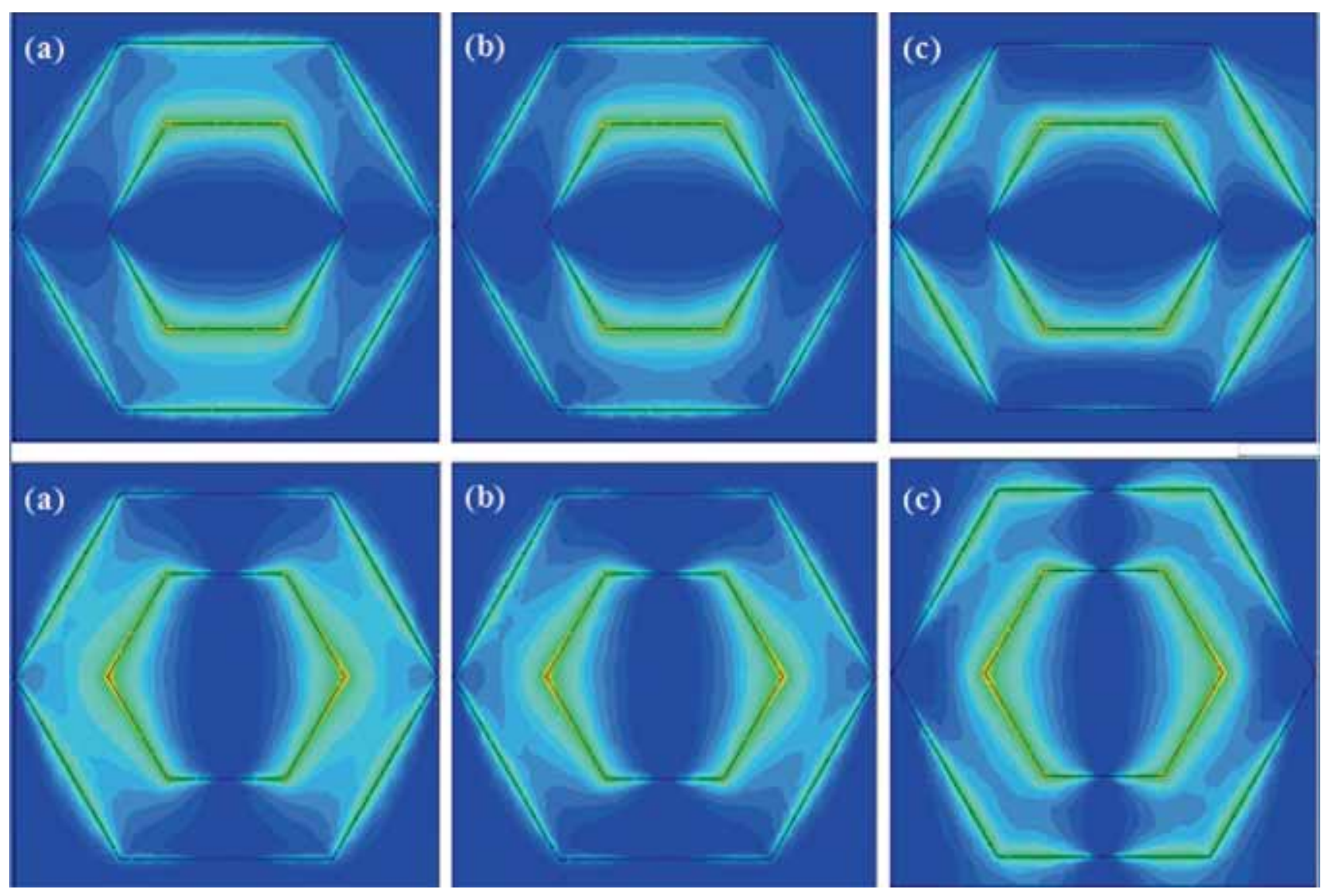

Figure 10. TE mode and TM mode electric field distribution at the three frequencies: (a) $0.2 \mathrm{THz}$, (b) $0.36 \mathrm{THz}$ and (c) $0.52 \mathrm{THz}$.

The obtained frequency band is from 0.26 to $0.52 \mathrm{THz}$. The magnitude plot of the transmission is shown in figure 4 .

Furthermore, the angle of incidence of the $\mathrm{THz}$ wave does not affect the polarization state of the hexagonal shaped FSS filter structure. On defining the polarization angle which is the angle between the direction of polarization and the $x$-axis of the coordinate, the polarization state of the designed FSS filter structure i.e., at the transverse electric (TE) mode and at the transverse magnetic (TM) mode, and the frequency response of the designed FSS filter coincide well with each other. Also, the angle of incidence has been varied from 0 to $45^{\circ}$ increasing in intervals of $15^{\circ}$ and found a concurrence in their frequency response. The obtained frequency spectra of the FSS filter structure at different modes and at different angles of incidence are noted and are plotted as shown in figures 5 and 6 , respectively.

The process of fabrication has then been focused and carried out using the femtosecond laser micromachining setup. The laser source for the process of micromachining is the Ti:sapphire laser emitting laser pulse in a wavelength of $800 \mathrm{~nm}$. The term ultrashort is coined as the laser source emitting laser pulses with a pulse duration of less than $100 \mathrm{fs}$. The XYZ-turning stage i.e., the object positioning system integrated along with the Galvano scanner, flip mirrors and a
CCD camera is synchronized in order to place the sample in position enabling it to be appropriately exposed to the laser beam. The focused beam diameter of the laser pulse is $20 \mu \mathrm{m}$ and a lens with a focal length of $100 \mathrm{~mm}$ has been used for focusing the beam on the sample where the microstructure is intended to be created. The attenuator and half-wave plate are used for the process of controlling the power of the incident laser beam. The scan rate is set as $40 \mathrm{~mm} \mathrm{~s}^{-1}$ and the repetition rate is $10 \mathrm{kHz}$ [13].

Before the fabrication process, the process of hatching has been performed and the hatched image of the designed FSS filter structure has been loaded in the laser micromachining setup system. The laser beam will follow the hatching lines and etch accordingly. The scanning electron microscopy image of the fabricated broadband FSS filter structure is depicted in figure 7 in which $300 \mu \mathrm{m}$ zooming of the sample which consists of the whole fabricated array i.e., periodic unit cells can be performed and obtained. Owing to the flow of the laser beam along the hatching lines, a slight toothed structural outcome has been obtained instead of a smooth straight finishing.

The measurement results of the fabricated FSS filter structure have been obtained using THz time domain spectroscopy. The plot of the measured result is depicted in figure 8. From 


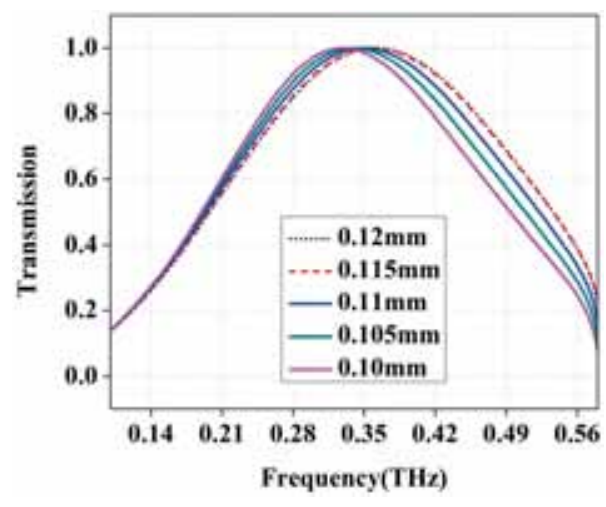

Figure 11. Transmission in $\mathrm{dB}$ for different widths of the hexagonal slot.

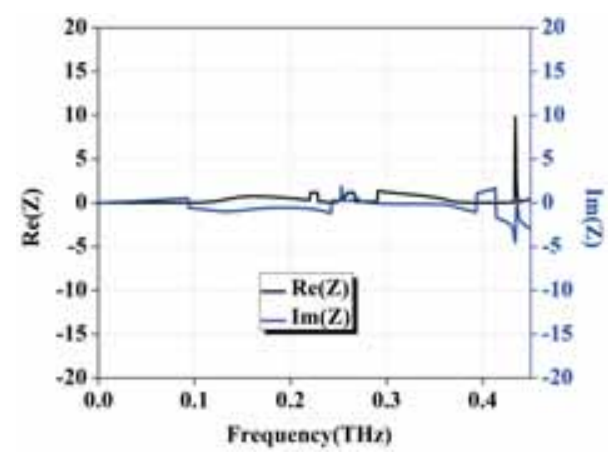

Figure 12. Impedance, $Z$ of the FSS structure.

the figure, it is evident that the measured and the simulated results, when compared, are in concurrence with each other showing the compatibility of the designed FSS filter structure.

Broadband frequency spectra covering the frequency range of $0.29-0.52 \mathrm{THz}$ are also achieved by fabrication. This frequency band covers two of the prominent prevailing applications of $\mathrm{THz}$ frequency band: MARSHALLS airborne limb sounder and European space agency (ESA). This design achieves a transmission of more than $97 \%$ as the conventional FSS filter invoked structure does not possess such a high-transmission rate. The full-width at half maximum is calculated as $400 \mathrm{GHz}$.

The TE mode and TM mode surface current distribution is depicted in figure 9. Similarly, the TE mode and TM mode electric field distribution of the FSS filter is depicted in figure 10 evidencing the hexagonal slot being responsible for the broadband resonance.

The width of the hexagonal shaped slot of the FSS filter structure has been varied in steps of $5 \mu \mathrm{m}$ from 100 to $120 \mu \mathrm{m}$. The optimum response of achieving a broadband frequency response with a bandwidth of $300 \mathrm{GHz}$ and the maximum transmission of $97 \%$ have been obtained at the width of $110 \mu \mathrm{m}$ and the plot is shown in figure 11 .

The impedance, $Z$ of the FSS filter structure, is shown in figure 12. From the graph, it is inferred that the real part of the effective impedance is more likely closer to unity $[\operatorname{Re}(Z) \approx$ 1] which matches the free space impedance and the imaginary part of the effective impedance is close to zero $[\operatorname{Im}(Z) \approx 0]$.

\section{Conclusion}

The broadband frequency response has been achieved in this paper by means of only invoking the FSS structure excluding the presence of ground plane and also excluding the multilayered FSS technique. A broadband frequency response of $0.4 \mathrm{THz}$ has been achieved only through the hexagonal geometry which is very much pragmatic in the $\mathrm{THz}$ frequency domain which has been evolving in the recent era. The two prevailed techniques of obtaining a broadband frequency response has been abrogated by this proposed simple hexagonal shaped FSS structure. The presented hexagonal shaped FSS filter structure offers angle-resolved polarization insensitiveness which presents it as a highly advantageous FSS structure to be invoked in spatial applications. Other prominent pros of this work are the coverage of the frequency bands of MARSHALLS limb sounder and ESA.

\section{References}

[1] Sanphuang V, Nahar N K and Volakis J L 2013 Natl. Aerosp. Electron. Conf. Proc. IEEE 18

[2] Qiao S, Zhang Y, Zhao Y, Zhou Y, Liang S and Yang Z 2016 IEEE Trans. Terahertz Sci. Technol. 6292

[3] Zhang X Y, Zheng Z X, Li X X, Tan R B, Zhang Z P, Zhou Y et al 2012 Adv. Mater. Res. 571362

[4] Ferraro A, Zografopoulos D C, Caputo R and Beccherelli R 2017 J. Sel. Top. Quantum Electron. 231

[5] Xia B G, Yao C F, Huang J, Meng J, Zhang D H and Zhang J S 2013 Electron. Lett. 491398

[6] Dickie R, Cahill R, Fusco V, Gamble H S and Mitchell N 2011 IEEE Trans. Terahertz Sci. Technol. 1450

[7] Sanphuang V, Ghalichechian N, Nahar N K and Volakis J L 2016 IEEE Trans. Terahertz Sci. Technol. 6583

[8] Zhang J L, Zhang Y, Gao K and Zhao G Z 2013 Chinese Sci. Bull. $\mathbf{5 8} 4687$

[9] Chen X and Fan W H 2015 Mater. Res. Express 21

[10] Kumar A and Kartikeyan M V 2013 Act. Passiv. Electron. Components 66

[11] Ebrahimi A, Nirantar S, Withayachumnankul W, Bhaskaran M, Sriram S, Al-Sarawi S F et al 2015 IEEE Trans. Terahertz Sci. Technol. 5761

[12] Manikandan E, Sreeja B S, Radha S and Bathe R N 2018 Mater. Lett. 229320

[13] Manikandan E, Sreeja B S, Radha S, Bathe R N, Jain R and Prabhu S S 2018 J. Infrared Millim. Terahertz. Waves 34489 